\title{
Ultrastructure des Diatomées de Nouvelle-Calédonie. Première partie.
}

\author{
R. Le Cohul
}

Mots clés : Diatomées, microscopie électronique, morphologie.

Dans cette première note, 13 taxons, dont 5 endémiques de Nouvelle-Calédonie, sont examinés au microscope électronique à balayage (M.E.B.) et au microscope électronique à transmission (M.E.T.). La liste des espèces observées est la suivante: Amphipleura guillauminii, Achnanthes lata, Brachysera neocaledonica var. angusta (nov. comb.), Cocconeis neocaledonica, Cymbella delicatula, Cymbella subturgida. Denticula elegans, Epithemia cistula, Navicula bryophila. Navicula viridula var. rostellata, Stenopterobia intermedia, Su rirella neocaledonica var. punctata. La structure de Cocconeis neocaledonica Maillard se révèle très originale.

Ultrastructure of the diatom flora from New-Caledonia. Part. I.

Keywords : Diatoms, electron microscopy, morphology.

This is the first contribution on the ultrastructure of diatoms recorded in New-Caledonia. Among the 13 taxa described, 5 are endemic. Of particular interest is the remarkable structure observed in the species Cocconeis neocaledonica Maillard,

\section{1. - Introduction}

Les diatomées d'eau douce de la NouvelleCalédonie ont fait l'objet de trois publications : Carter N. (1922), Manguin (1962) et Maillard (1978). Manguin avait décrit 38 nouvelles espèces, Maillard 78 nouveaux taxons. Aucun de ces auteurs n'ayant donné de description en microscopie électronique, il m'a semblé utile d'entreprendre une telle étude en portant une attention particulière aux espèces endémiques ou encore rarement observées.

\section{2. - Observations et discussion}

Amphipleura guillauminii Manguin (fig. 1/2/3/4/ 5/36).

Manguin 1962 : pl. VIII, fig. 3.

\footnotetext{
1. Laboratoire d'Hydrobiologie, U.A. 695 C.N.R.S., Université P. Sabatier, 118 route de Narbonne, 31062 Toulouse Cedex (France). Ce travail a été réalisé avec la collaboration technique de Mme J. Brabet.
}

Cette espèce endémique, aisément reconnaissable grâce au rétrécissement de la valve au niveau des pores centraux, a été également signalée par Maillard (1978, pl. VIII, fig. 2). En ce qui concerne les aréoles, le M.E.T. (fig. 36) confirme les observations antérieures (Cox 1975, Gerloff \& Helmcke 1977). Au M.E.B., en vue interne ( $\mathrm{fig} .3 / 4 / 5$ ), chaque nodule terminal, assimilé par Schoeman \& Ashton (1982) à un hélictoglosse modifié, se prolonge, de chaque côté du raphé et ce jusqu'au nodule central, par des bourrelets fortement silicifiés; ceux-ci se réunissent ensuite en une nervure axiale qui correspond à ce que l'on peut appeler le nodule central. La structure interne de $A$. guillauminii, qui n'est pas sans rappeler celle du g. Frustulia, est tout à fait comparable à celle de A. pellucida Kütz. (Cox 1975, Schoeman \& Ashton 1982). Par contre, en vue externe, A guillauminit s'en différencie par la fissure terminale incurvée des 2 branches du raphé ( $f i g .1 / 2 / 36$ ); ce caractère rapproche cette espèce du g. Berkeleya (Cox 1975) mais il ne semble pas suffisant pour l'y inclure. 
Achnanthes lata Hustedt (fig. 37/38).

Hustedt 1942 : fig. 38/43.

Cette espèce décrite par Hustedt aux Iles Célèbes a été retrouvée en Nouvelle.Calédonie par Manguin (1962) et Maillard (1978). Au M.E.T. (fig. 37/38), on retrouve les caractères déjà signalés au microscope photonique: stries plus écartées dans la partie médiane, fissure terminale du raphé légèrement incurvée, léger élargissement de l'aire axiale au niveau de l'aire centrale sur les 2 valves. Les ponctuations des stries sont bacilliformes.

Brachysera neocaledonica (Maillard) nov. comb. var. angusta (Maillard) nov. comb. (Syn : Anomoeoneis neocaledonica Maillard var. angusta Maillard) (fig. 6/7/39/40).

Maillard 1978 : pl. II, fig. 5.

Round \& Mann (1981) ont montré que la plupart des espèces, notamment d'eau douce, du genre $A$ nomoeoneis devaient être transférées dans le g. Brachysera; c'est le cas de Anomoeoneis neocaledonica var. angusta. Cette variété présente les principaux caractères du g. Brachysera, que ce soit au M.E.T. ou au M.E.B. : face valvaire portant des mamelons sur les interstries (fig. 6/7); stries constituées d'aréoles allongées transapicalement (fig. 39/40) interrompues sur les bords valvaires par une saillie fortement sili. cifiée (fig. 7); sur le manteau, stries et interstries ont le même aspect que sur la face valvaire; raphé placé dans une cannelure, à bordures épaisses, qui s'interrompt au niveau de l'aire centrale.

Cocconeis neocaledonica Maillard (fig. 8/9/10/11/ 12/13/14/15/16/17/43).

Maillard 1978 : pl. II, fig. 1.

Ce Cocconeis a une structure très particulière qui présente de grandes analogies avec celle d'espèces marines (Ricard, comm. pers.).

Au M.E.B., la valve à raphé (fig. 8/9) a pu être observée seulement en vue externe : courtes stries marginales interrompues par un sillon longitudinal, puis reprenant sous la forme d'une simple ponctuation; entre cette zone marginale et l'aire axiale, toute ornementation semble absente sur la face valvaire. De chaque côté des 2 branches du raphé, on observe la présence d'une rangée de ponctuations. Au M.E.T. (fig. 43), la seule observation réalisée, si on l'interprète comme une vue interne, semblerait montrer la présence de stries dans la partie de la face valvaire dépourvue d'ornementation en vue externe. I] faut noter que la partie centrale de la face valvaire semble peu silicifiée et se brise facilement.

La valve sans raphé, en vue externe (fig. 11/12) présente des stries à ponctuations ayant souvent l'aspect d'un $\chi$ (velum à volae ?). En vue interne, il faut remarquer que les côtes séparant les stries s'atténuent dans l'espace compris entre les deux dernières rangées d'aréoles au bord de l'aire axiale (fig. 13 . flèche) : cette zone moins silicifiée constitue sans doute la zone de fracture donnant naissance à l'ou. verture observée chez de nombreux spécimens.

L'originalité de cette espèce réside dans la présence de 2 cloisons internes parallèles aux faces valvaires. Sous l'hypovalve, une première cloison interne (fig. 10) présente de grandes similitudes avec la valve à raphé, tout au moins au niveau de ce que l'on pourrait appeler aire axiale qui porte, de chaque côté de la réplique du raphé, 2 rangées de petites protubćrances ; la partic correspondant à l'aire centrale est toujours marquée par la présence d'une ouverture. A partir des bords de la valvocopula se détachent des arcs-boutants s'élargissant latéralement pour se réunir et former une plate-forme ; celle-ci, limitée par une cannelure longitudinale, est ornée d'une série de crêtes dans l'alignement des protubérances de l'« aire axiale ». Les arcs-boutants laissent entre eux des ouvertures (pseudoloculae de Maillard 1978).

Sous l'épivalve, la cloison interne (fig. 14/15) se révèle être presque un duplicata de la valve sans raphé ; la différence réside dans le fait que les côtes séparant les lignes de pores sont ici seulement marginales (fig. 14). Sur les parois de la valvocopula s'insèrent des supports côtelés à leurs bases et évasés vers l'intérieur du frustule ; ils laissent entre eux ce que Maillard (1978) a appelé des pseudoloculae (fig. 16/17). Des ouvertures de forme irrćgulic̀re (fig. 15/16) ont souvent été observées dans la partie axiale de la cloison. Il n'a pas été possible de déterminer avec certitude :

1) si ces ouvertures sont dues à des cassures au niveau de la zone "sans côte" de la cloison qui serait moins silicifiée (bien qu'aucun débris n'ait été détecté au cours de nos investigations, microscope optique ou électronique) ;

2) si elles sont le résultat d'un processus naturel observé seulement chez certains individus. Cette 
structure avec double cloisons internes n'est pas sans rappeler celle du g. Diatomella, mais dans le cas présent elle est beaucoup plus élaborée.

\section{Cymbella delicatula Kütz (fig. 44/45/46).}

Les spécimens rencontrés en Nouvelle-Calédonie correspondent parfaitement au schéma proposé par Cox (1977, fig. 10). Au M.E.T. (fig. 44/45), on retrouve les caractères de l'espèce : raphè à longue fissure terminale orientée vers le bord dorsal, appartenant au groupe "Revers-Lateralraphé " défini par Krammer (1979, pl. 3, fig. 22), stries aux ponctuations orientées obliquement (Cox 1977) donnant même parfois l'impression d'une torsade (fig. 46, flèche). Ce dernier caractère distingue nettement $C$. delicatula de C. microcephala (Schoeman \& Ashton 1983) et de C. cesatii (Germain 1981, a); de plus, comme le montrent les illustrations des auteurs précités, les stries de ces deux dernières espèces ont un aspect cannelé que l'on ne retrouve pas chez $C$. delicatula. Chez cette espèce, Cox (1977) signale la présence de stigmas à l'extrémité des 3 stries centrales du bord ventral; dans le cas des spécimens de NouvelleCalédonie, ces stigmas sont en général au nombre de 4 (fig. 44/46).

Cymbella subturgida Hust. var. neocaledonica Manguin (fig. 18/19).

Manguin 1962 : pl. V, fig. $7 \mathrm{a} / \mathrm{b}$.

La structure de ce taxon est déjà bien visible en microscopie optique avec un raphé à ligne d'accompagnement (Lateralraphé de Krammer 1979), fissure terminale dirigée ventralement. Le M.E.B., vue externe (fig. 18/19), montre que la fissure apparaissant ventralement au microscope optique correspond à la fente des 2 branches du raphé, la ligne d'accompagnement dorsale à la structure du raphé dans la face valvaire. Les stries (intercostae) sont constituées, si l'on adopte la terminologie de Krammer (1982), par des foraminae qui se prolongent sur le manteau.

\section{Denticula elegans Kütz (fig. 20).}

Espèce observée seulement en vue externe. Face valvaire traversée de part en part par le raphé avec rangées régulières d'aréoles.
Epithemia cistula (Ehr.) Ralfs (fig. 21/22/23/24).

Au M.E.B., la vue externe d'un frustule ( $f i g .21$ ) confirme la remarque de Sims (1983), à savoir que la largeur de la ceinture diminue d'un pôle à l'autre. Au cours de nos investigations, seules des vues internes de valves associées à leurs valvo-copulae très étroites ont pu étre observées (fig. 22/23/24). Les cótes primaires (« costate fibulac") déterminent une seule rangée d'aéroles; elles peuvent parfois fusionner à différents niveaux ; on note aussi la présence de côtes secondaires (virgae) s'interrompant au canal raphéen. Celui-ci est en relation avec l'intérieur de la valve par l'intermédiaire de portulae et se termine aux pôles par une ouverture triangulaire où l'on peut apercevoir un petit hélictoglosse ( $f$ ig. 24, flèche).

\section{Navicula bryophila J.b. Petersen (fig. 42).}

Cette espèce a été identifiée comrne étant $N$. bryophila, en parfait accord avec la figure (pl. 6, fig. 5) de Coste et Ricard (1982); elle présente de grandes analogies avec $N$. maillardii Germain (1982, pl. 4, fig. 20) décrite aux îles Kerguelen : nombre de stries équivalent avec brusque changement d'inclinaison et bifurcation au niveau où les bords de la valve s'incurvent vers les pôles, même type de structure du raphé avec inflexion des 2 branches du raphé vers le nodule central et fissures terminales longues et recourbées vers l'arrière. La structure fine des aéroles (Coste et Ricard 1982, pl. 8, fig. 10 ; Germain 1982, pl, 4, fig. 23) est pratiquement identique. Généralement, les spécimens de $N$. maillardii aux îles Kerguelen sont tri-ondulés et plus ou moins capités ; les exemplaires de $N$. bryophila de Nouvelle-Calédonie ou de l'île Maurice (Coste et Ricard 1982) sont plutôt rostrées avec des bords valvaires parallèles ou légèrement convexes. $N$. maillardii a été retrouvée en République Sud-africaine par Schoeman \& al. (1984). Ces auteurs soulignent également les analogies entre les deux espèces précitées. Si l'une de leurs illustrations ( $f i g .51$ ) répond parfaitement au type de $N$. maillardii tel que le définit Germain (1982); une autre (fig. 52), bien que plus convexe dans sa partie centrale et légèrement tri-ondulée, se rapproche beaucoup de l'espèce identifiée comme étant $N$. bryophila en Nouvelle-Calédonie ou à l'île Maurice. Il faut également remarquer que, sur certains dessins de Hustedt (1961/66), fig. 1237), une légère ondulation des bords valvaires chez 
N. bryophila peut être décelée. On peut donc se demander si, avec les variations inherentes a tout taxon en fonction du milieu où il vit, $N$. bryophila et $N$. maillardii ne sont pas une seule et même espèce.

Navicula confervacea (Kütz.) Grun. (fig. 25/26/27).

Nos observations confirment les études antérieures (Coste 1975, Schoeman \& Archibald 1980) : présence d'épines sur les bords marginaux des valves (fig. 26) permettant l'assemblage des frustules en colonies ; branches du raphé se terminant souvent par des boucles fermées ( $f$ ig. 27) au niveau de l'aire centrale; aéroles bacilliformes.

\section{Navicula viridula Kütz. var. rostellata (Kütz.) Cleve (fig. 41).}

Ni l'espèce, ni cette variété n'ont été rencontrées en Nouvelle-Calédonie par Manguin (1962) et Maillard (1978); par contre, ce dernier signale la présence de la var، capitata Mayer (Germain 1981 b, l'assimile au type de Kützing). La variété rostellata se présente telle que la définit Germain (1981 b) avec notamment l'asymétrie nette de l'aire centrale et la bifurcation des stries d'un seul côté environ à la moitié de chaque demie valve. Ces caractères sont également bien visibles sur les illustrations de LangeBertalot (1980), en particulier la bifurcation des stries ; par contre, Patrick \& Reimer (1966) ne signalent pas ce caractère dans leur description (ils le figurent seulement sur une moitié de valve, pl. 48 , fig. 12).

\section{Stenopterobia intermedia Lewis (fig. 28/47).}

Cette espèce demande de nouvelles investigations, notamment au niveau du canal raphéen. Au M.E.B. (fig. 28), les côtes élargies vers les bords larvaires et s'amincissant vers l'aire axiale semblent ponctuées comme le montre l'illustration au M.E.T. (fig. 47); sur cette dernière figure, on note la présence de portulae (flèche).

Surirella neocaledonica Manguin var. punctata Maillard (fig. 29/30/31/32/33/34/35).

Maillard: pl. VI, fig. 10.

L'espèce et la variété ont été décrites en NouvelleCalédonie. Seules des vues externes ont pu être observées au M.E.B. Les canaux alaires se prolongent sur la face valvaire sous la forme d'ondulations étroites ( $\mathrm{fig} .33 / 34$ ) ; ils sont percés de deux groupes de 2 rangées de pores (fig. 31) fusionnant en une seule ligne sur les ondulations; sur celles-ci, comme le signale Maillard (1978), se placent 2 ou 3 épines (rarement 1). Entre les canaux alaires, les fenêtres à barres fenestrales ( $\mathrm{fig}$. 30/31/32/33) sont tapissées intérieurement de lignes de pores convergeant vers les bords valvaires (fig. 35) (seulement visibles sur les spécimens fortement attaqués à l'acide); extérieurement, ces lignes de pores se prolongent au sein des dépressions entre les ondulations ( $f$ ig. 31/32). La partie axiale de la valve est marquée par un bombement sur lequel, de chaque côté, en alternance régulière, s'insèrent les ondulations (fig. 33/34). Sur ce bombement, on note la présence de pores irrégulièrement disposés (fig. 31, flèches étêtées).

\section{Travaux cites}

Carter (N.). 1922. - Inventaire des algues d'eau douce de la Nouvelle-Calédonie. Joumal Linn. Soc, 46:44-68.

Coste (M.). 1975. - Sur la prolifération dans la Seine d'une diatomée benthique tropicale. Navicula confervacea (Kütz.) Grunow. Annls Limnol., 11 (2): 111-123.

Coste (M.) \& Ricard (M.). 1982. - Contribution à l'étude des diatomées d'eau douce des Seychelles et de lîle Maurice. Cryptogamie, Algologie, III (4) : 279-313.

$\operatorname{Cox}($ E.). 1975. - A reappraisal of the diatom genus Amphipleura Kütz, using light and electron microscopy. Br. phycol. J., 10 : 1-12.

Cox (E.). 1977. - Variation in valve structure between species of the diatom genus Cymbella. C.A. Ag. Nova Hedwigia, 28: $427-449$.

Gerloff (J.) \& Helmcke (J.G.). 1977. - Diatomeenschalen im Elektronen-mikroskopischen. Bild, Teil X, Cramer, Vaduz : pl. 924/1023.

Gemain (H.). 1981 a. - Navicula falaisiensis Grunow, Cymbella cesatii (Rabh.) Grul. et var. capitata nov. var., Cymbella microcephala Grun. et Cymbella delicatula Kützing. Cryptogamie, Algologie, II (2) : 123-129.

Germain (H.) 1981 b. - Flore des Diatomées, eaux douces et saumâtres. Boubée, Paris : $\mathbf{4 4 4}$ p.

Germain (H.). 1982. - Quatre Navicules du groupe des Subtilissimae (Diatomophyceae). Cryptogamie, Algologie, III (2) : 105-111

Hustedt (F.). 1942. - Susswasser-Diatomeen des Indomalayischen Archipels und Hawai-Inseln. Intemat. ges. Hydrobiol " Hydrogr., 42 (1/3) : 1-252.

Hustedt (F.). 1961/1966. - Die Kieselalgen. In: Rabenhorst 4. Kryptogamen-Flora won Deutschland, Osterreich und der Schweiz, Bd VII, T. 3, Lief. 1/4, Leipzig : $816 \mathrm{p}$.

Krammer (K.). 1979. - Zur Morphologie der Raphe bei der Gattung Cymbella. Nova Hedwigia, 31 (4) : 993-1029.

Krammer (K.). 1982. - Valve morphology in the genus Cymbella C. A. Agardh. In micromorphology diatoms valves, Helmcke J.G. \& Krammer K., édit., vol. XI, Cramer, Vaduz: 299 p.

Lange-Bertalot (H.). 1980. - Zur taxonomischen Revision einiger ökologisch wichtiger * Naviculae lineolatae . Cleve. Die Formenkreise um Navicula lanceolata, $N$. viridula, $N$. cari. Cryptogamie, Algologie, I (1) : 29-50.

Maillard (R.). 1978. - Contribution à la connaissance des diatomées d'eau douce de la Nouvelle-Calédonie (Océanie). Cah. O.R.S.T.O.M., sér. Hydrobial, XII (2) : 143-172. 
Manguin (E.). 1962. - Contribution à la connaissance de la flore diatomique de la Nouvelle-Caledonie. Mém. Mus. Hist. Nat., Paris, nouv. sér, B, Bot., XII (1) : 1-40.

Patrick (R.) \& Reimer (C.W.). 1966. - The Diatoms of the United States, vol. 1, Acad. Nat. Sci. Philadelphia, Monog. 13:668 p.

Round (F.E.) \& Mann (D.G.). 1981. - The diatom genus Brachysera. 1. Typification and separation from Anomoeoneis. Arch. Protistenk. 124:221-231.

Schoeman (F.R.) \& Archibald (R.E.M.). 1980. - The diatom flora of Southern Africa, $n^{\circ} 6$

Schoeman (F.R.) \& Ashton (P.J.). 1982. - The diatom flora in the vicinity of the Pretoria Salt Pan, Transvaal, Republic of South Africa I. Nova Hedwigia, 73 : $21-54$.
Schoeman (F.R.) \& Ashton (P.F.) 1983. - The diatom flora in the vicinity of the Pretoria Salt Pan, Transvaal, Republic of South Africa. Part II. S. Afr. J. Bot., 2: 191.201.

Schoeman (F.R.), Archibald (R.E.M.) \& Ashton (P.J.). 1984. - The diatom flora in the vicinity of the Pretoria Salt Pan, Transvaal, Republic of South Africa. Part III (final). S. Afr. J. Bot., 3: 191.207.

Sims (P.A.). 1983. - A taxonomic study of the genus Epithemia with special reference to the type species E. turgida (Ehrenb.) Kütz. Bacillaria, 6 : 211-235. 


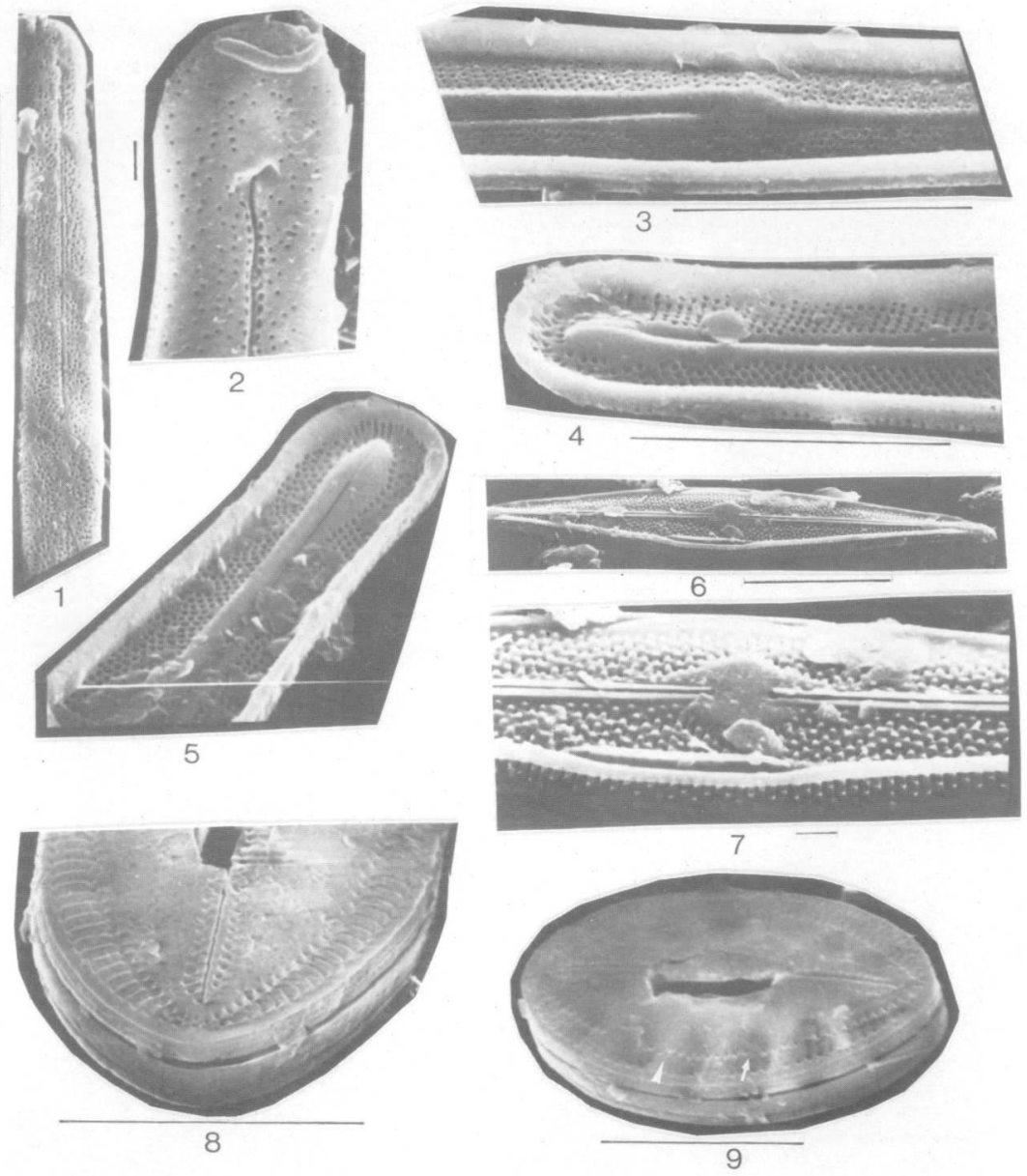

Planche I. - (microscopie à balayage). Echelle $10 \mu$ sauf fig. 2 et $7,1 \mu \mathrm{m}$.

Amphipleura guillauminii, 1 : vue externe ; 2 : vue externe de l'extrémité de la valve, fissure terminale du raphé incurvée ; 3 : vue interne montrant la fusion des bourrelets bordant le raphé pour ensuite donner une côte axiale ; 4/5: vues intermes de l'extrémité de la valve montrant la terminaison du raphé. Brachysera neocaledonica var. angusta, 6 : vue externe; 7 : détail de l'aire axiale et du manteau. Cocconeis neocaledonica, 8 : valve à raphé - vue externe : 9 : vue légèrement oblique d'une valve à raphé ; ouvertures latérales (pseudoloculae) de la cloison interne décelables (flèche) ainsi que les arcs-boutants (flèche étêtée). Sur les fig. 8/9, noter la cassure au niveau de l'aire centrale coïncidant avec l'ouverture de la cloison interne. 

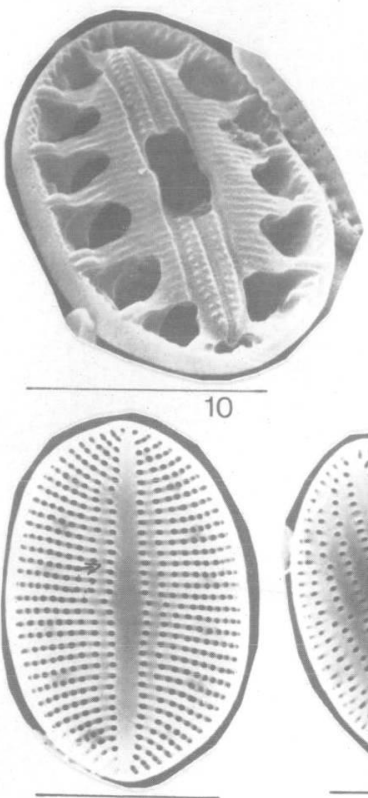

13

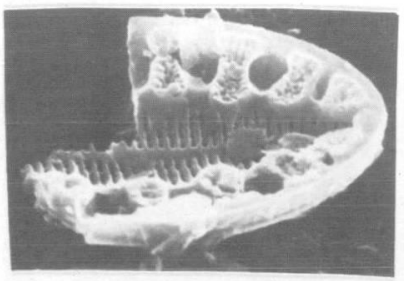

17

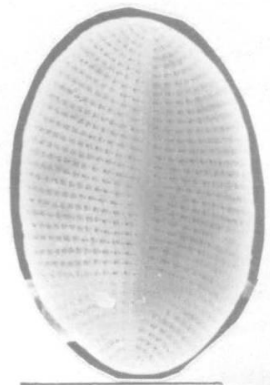

11

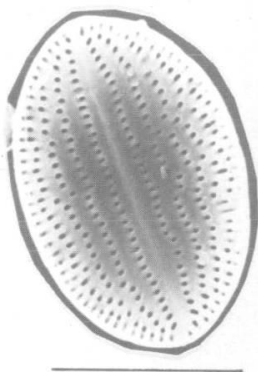

14
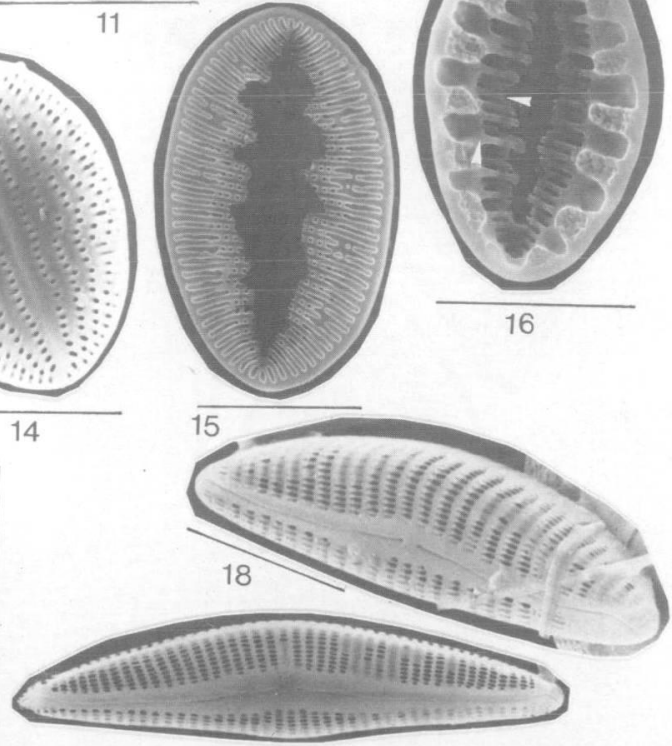

19

Planche II. - (microscopie à balayage). Echelle $10 \mu$

Cocconeis neocaledonica, 10 : vue de la cloison interne placée sous la valve à raphé ; 11 : valve sans raphé, vue externe : 12 : détail des stries de la fig. $11 ; 13:$ valve sans raphé, vue interne. Noter l'at ténuation des côtes (flèche); $14:$ vue de la cloison interne située sous la valve sans raphé (sans doute vue interne). Noter les côtes marginales : $15:$ vue externe? de la même cloison interne ; 16 : vue montrant les supports insérés sur la valvocopula. Les flèches montrent deux niveaux de côtes, l'une celles de la cloison interne, l'autre celles de la valve sans raphé. Noter sur les fig. 15/16 la forme irrégulière de l'ouverture axiale : 17: autre vue montrant les supports latéraux insérés sur la valvocopula. Cymbella subturgida var. neocaledonica, 18 ; vue externe légèrement oblique ; 19 : vue externe. 


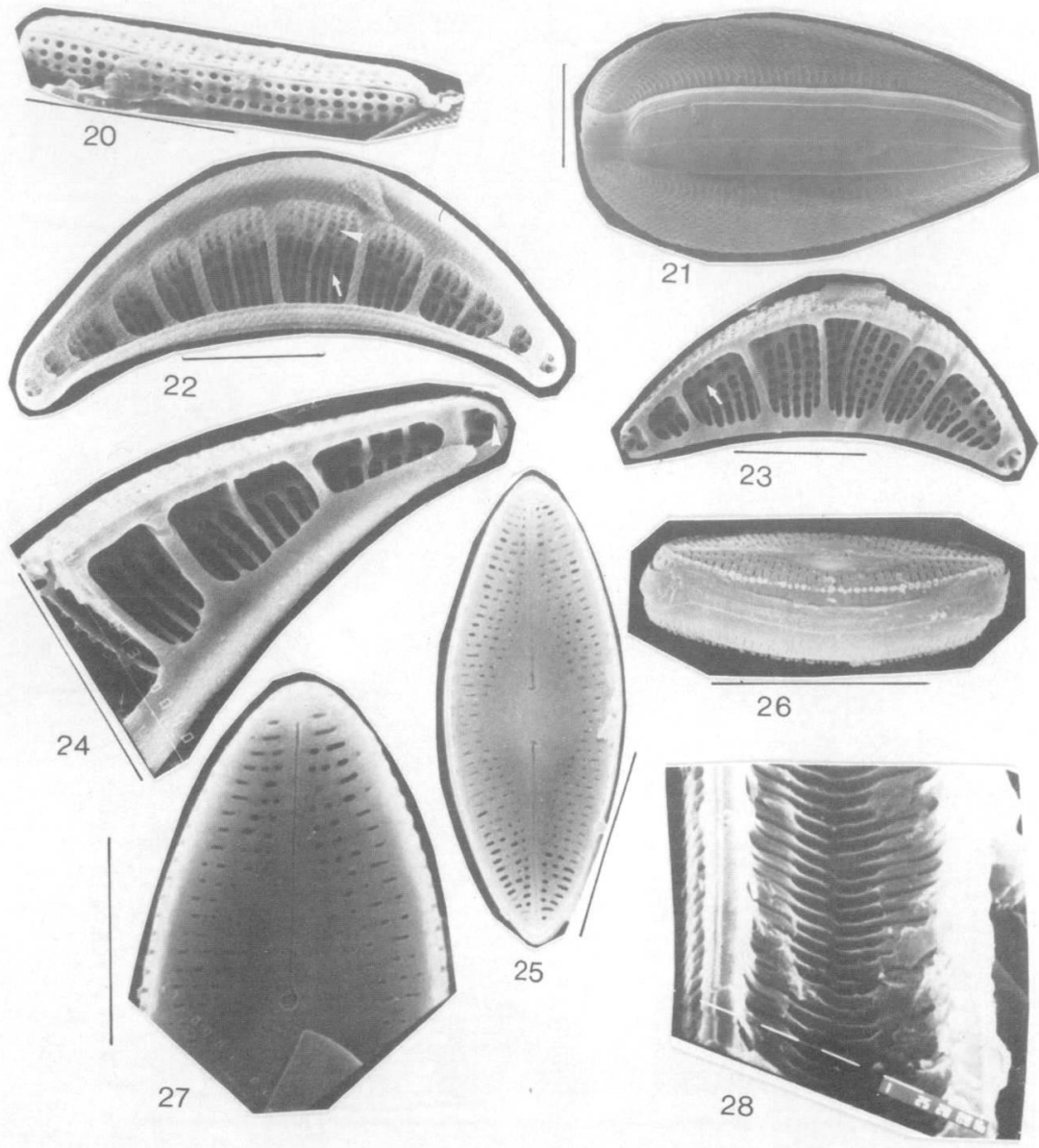

PlanChe III. - (microscopie à balayage). Echelle $10 \mu \mathrm{m}$ sauf fig. $27,5 \mu \mathrm{m}$.

20 : Denticula elegans, vue externe. Epithemia cistula, 21 : frustule entier ; 22 : vue interne avec valvocopula - côtes primaires (costate fibulae, flèche), côtes secondaires (virgae, flèche étêtée), canal raphéen -; 23 : vue interne avec valvocopula, canal raphéen et petit hélictoglosse (flèche étêtée). Navicula confervacea, 26 :frustule entier, épines marginales; $25 / 27$ : vues externes; 28 : Stenopterobia intermedia. 


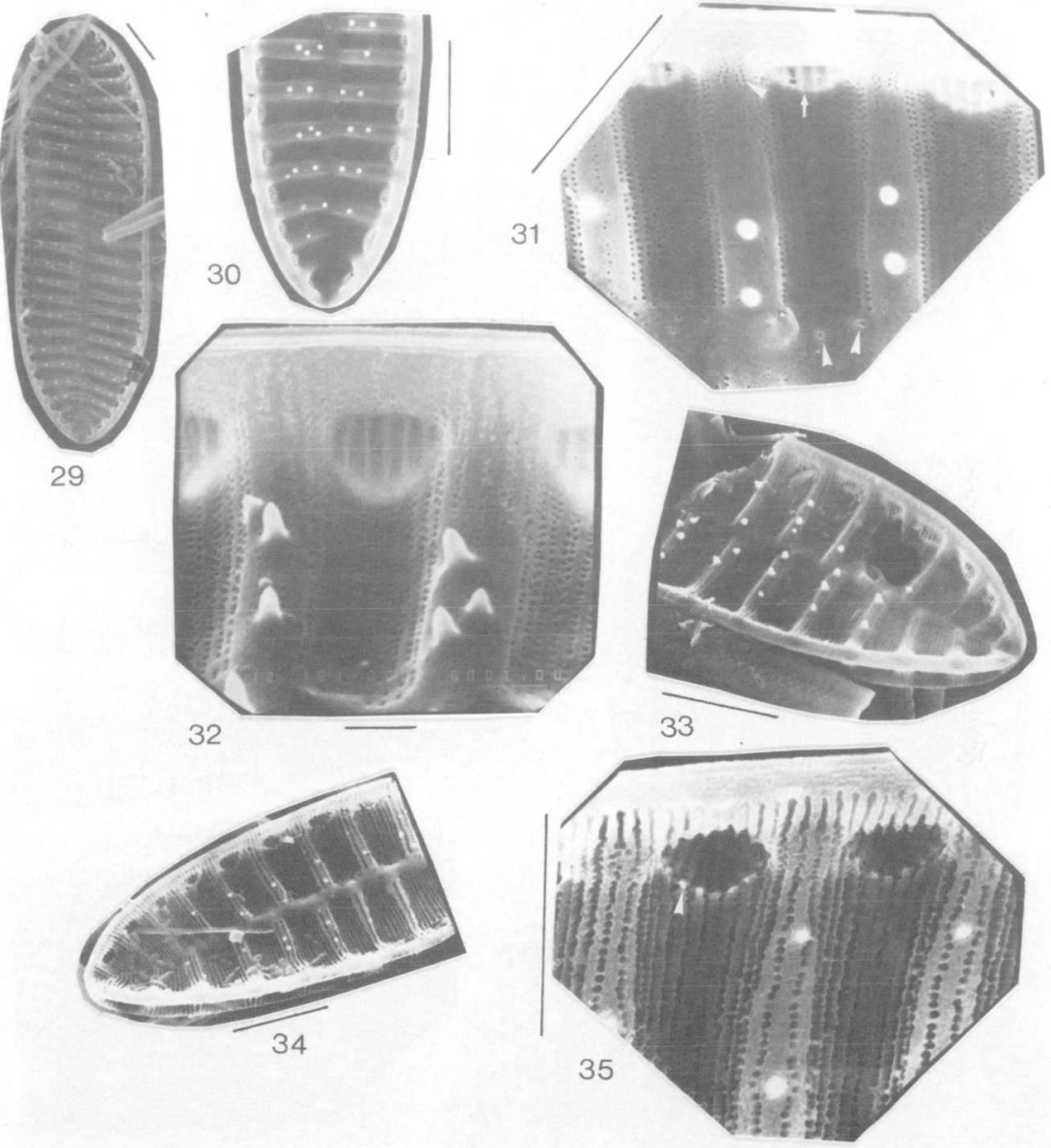

Planche IV. - (microscopie à balayage). Echelle $10, \mathrm{~m}$ sauf fig. $32,1 \mu \mathrm{m}$.

Surirella neocaledonica, $29 / 30$ : vues externes; 31 : détail de la fig. 30, canaux alaires (flèche étêtée), fenêtres à barres fenestrales (flèche), pores (flèches étêtées); 32 : même vue que la fig. 31 après rotation; $33 / 34$ : vues externes de valves fortement attaquées à l'acide ; 35 : détail de la fig. 34 montrant le départ des barres fenestrales (flèche étêtée) et lignes de pores à l'intérieur des fenêtres. 

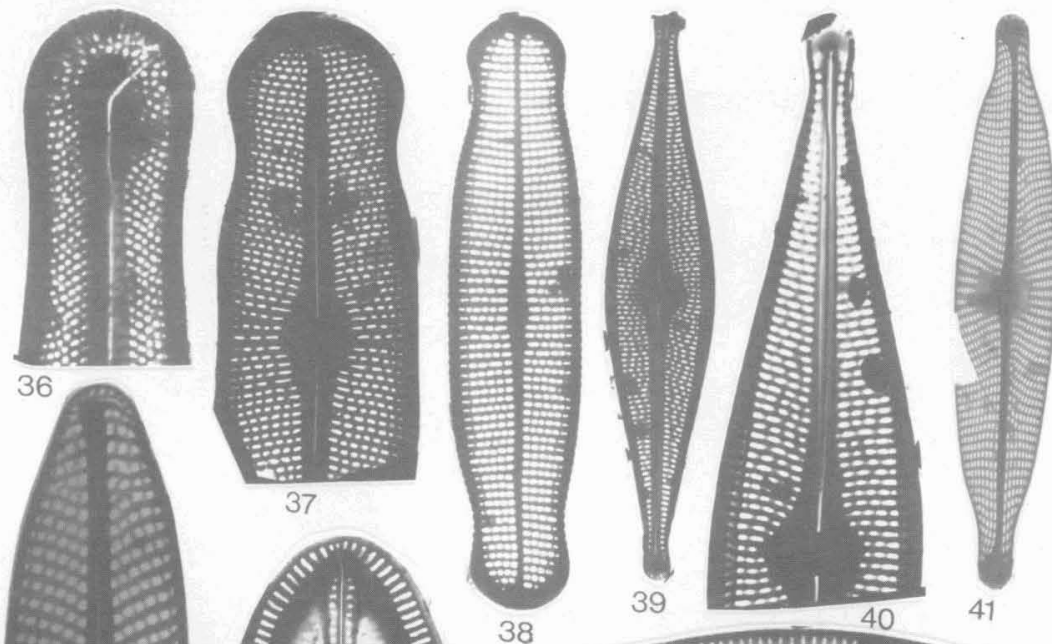

41

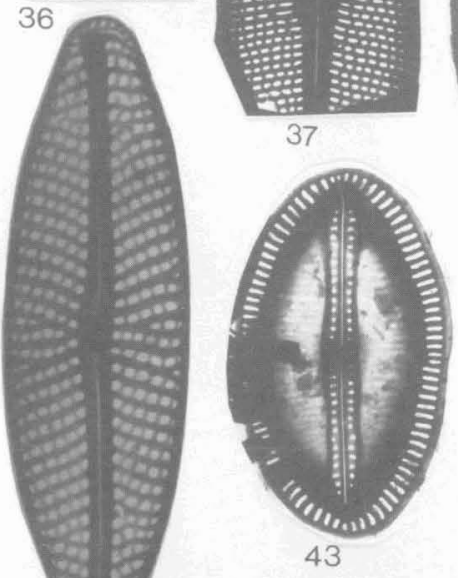

40

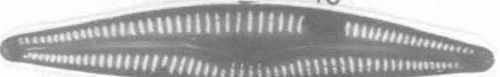
44
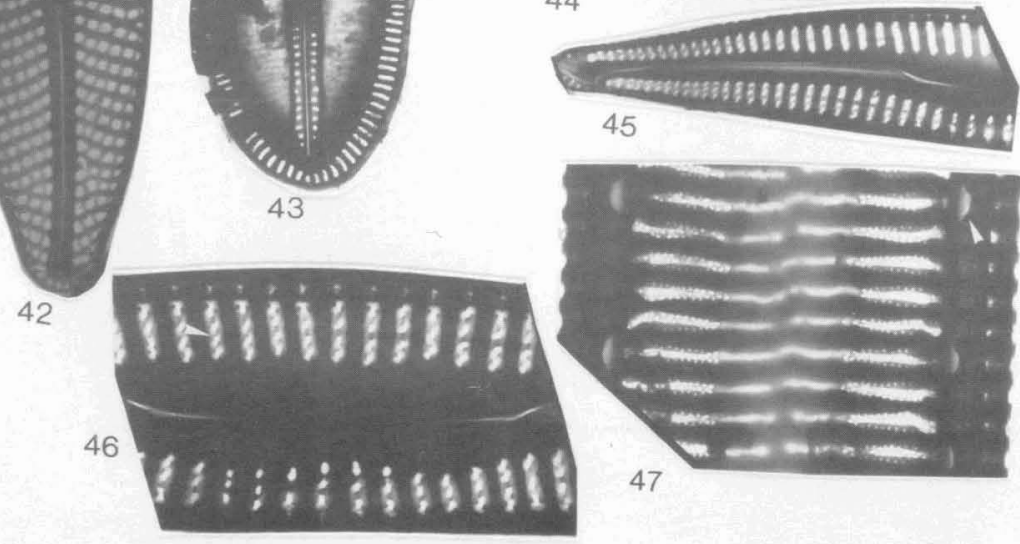

Planche V. - (microscopie à transmission).

36 : Amphipleura guillauminii \#4000. Achnanthes lata, 37 : valve à raphé \# $4000: 38$ : valve sans raphé \#2000. Brachy sera neocaledonica, $39:$ \# $2000 ; 40:$ \# $4000 ; 41:$ Navicula viridula var. rostellata \# 1600, 42 : Navicula bryophila \#4000. 43 : Cocconeis neocaledonica \# 2000. Cymbella delicatula, $44: \# 2000 ; 45: \# 4000 ; 46: \# 8000 ; 47:$ Stenopterobia intermedia, Portulae (flèche étêtée), \# 8000 . 